\title{
Incidencia y prevalencia de candidemia en pacientes críticamente enfermos en Colombia
}

\author{
Jorge A. Cortés, Jesús A. Jaimes y Aura L. Leal
}

\section{Incidence and prevalence of candidemia in critically ill patients in Colombia}

Introduction: Candidemia is an infectious complication with high morbidity and mortality in intensive care patients. Objective: The aim of this study was to determine the incidence and prevalence of candidemia in critically ill patients in Colombian hospitals between 2004 and 2008. Materials and Methods: Data from microbiologically confirmed candidemia cases, ICU stay and admissions were retrospectively recorded in 7 Colombian hospitals between 2004 and 2008. Time series analysis was performed with monthly incidence (number of cases of candidemia in relation to the number of patient-days) and prevalence (number of cases of candidemia in relation to the number of admissions) for each institution and the whole group. Results: 382 cases of candidemia were identified, with an incidence of 2,3 cases per 1.000 patient-days in ICU, and a prevalence of $1,4 \%$. There was a trend to increased incidence $(0,0066$ additional cases per $1.000 \mathrm{ICU}$-days per month) and prevalence $(0,0016$ additional cases por 100 patients per month) of candidemia. This increase of candidemia cases was due to a rise of non- albicans Candida species, which corresponded to $44 \%$ of total isolates. Discusion and Conclusions: Candidemia cases in colombian ICUs are increasing, especially those caused by non albicans Candida species.

Key words: Candidiasis, Candidiasis/epidemiology, Fungemia/epidemiology, Hospitals, Cross infection, incidence, prevalence, Intensive Care Units/statistics \& numerical data*

Palabras clave: Candida, candidiasis, candidiasis/epidemiología, candidiasis/microbiología, infección hospitalaria, incidencia, prevalencia, unidades de cuidados intensivos/estadística, datos numéricos.

\section{Introducción}

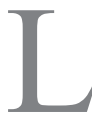

a candidemia es una complicación infecciosa de alta morbilidad en pacientes en unidades de cuidado intensivo (UCI), convirtiéndose en un problema emergente en los últimos años. Existen diversos reportes describiendo a las especies de Candida entre los cinco aislados más frecuentes en hemocultivos en infecciones intrahospitalarias en Estados Unidos de América (E.U.A.) y países desarrollados ${ }^{1}$. Su incidencia varía entre 0,035 y $0,15 / 1.000$ pacientes/día de estancia hospitalaria (DEH), en series norteamericanas y europeas ${ }^{2-4}$. En Colombia se encontró en el quinto lugar, con 5,2\% de hemocultivos positivos en $\mathrm{UCI}^{5}$.

La candidemia se asocia a una alta mortalidad en los pacientes críticamente enfermos. Se han reportado tasas de mortalidad atribuible entre 47 y 59\%, según diversas series, con los pacientes con algún tipo de inmunocompromiso, cirugías abdominales y con nutrición parenteral, los que presentan mayor letalidad ${ }^{6}$. Históricamente, Candida albicans ha sido el microorganismo más frecuentemente aislado, con una mortalidad atribuible que oscila entre 38 y $52 \%{ }^{7-9}$. Sin embargo, estudios recientes reportan un aumento en el número de casos de Candida no albicans asociado a resistencia a azoles ${ }^{8,10}$.
Existen diversos estudios realizados en Europa y E.U.A., sobre la incidencia y prevalencia de candidemia en distintos hospitales y UCIs; no obstante, la información en Latinoamérica es limitada. El objetivo de este estudio fue determinar la incidencia y prevalencia de candidemia en pacientes críticamente enfermos en Colombia entre 2004-2008 en siete instituciones.

\section{Materiales y Métodos}

\section{Tipo de estudio}

Estudio retrospectivo de series de tiempo de la incidencia de candidemias en las UCI de instituciones de tercer nivel de atención de Colombia.

\section{Población}

Todos los pacientes internados en las UCI de siete instituciones de tercer nivel de atención. En esta población se identificaron aquellos que presentaron candidemias validadas por el Comité de Infecciones Intrahospitalarias institucional en base a aislados microbiológicos en muestras de sangre. En Bogotá, a partir del año 2006, se unificaron las recomendaciones de las condiciones de obtención de muestra en instituciones hospitalarias ${ }^{11}$.
Universidad Nacional de Colombia, Bogotá, Colombia. Facultad de Medicina

Grupo de Investigación en Enfermedades Infecciosas (JAC). Departamento de Medicina Interna (JJ) Departamento de Microbiología (ALL).

Declaración de conflictos de interés: Jorge A. Cortés y Aura Lucía Leal han recibido apoyos de investigación para diferentes proyectos y para viajes de Pfizer $y$ Merck Sharp \& Dohme. Jesús Jaimes no tiene conflicto de interés alguno que declarar. Este proyecto fue financiado por División de Investigación de la Sede Bogotá y por la Facultad de Medicina de la Universidad Nacional de Colombia, a través de la convocatoria para apoyo a la investigación en salud 2010, código 11782.

Recibido: 1 de febrero de 2013 Aceptado: 14 de septiembre de 2013

Correspondencia a: Jorge Alberto Cortés jacortesl@unal.edu.co 


\section{Período de estudio}

Enero de 2004 a diciembre de 2008.

\section{Muestra}

Para el modelamiento de series de tiempo (metodología Box Jenkins) se tomó información de 60 períodos.

\section{Recolección de datos}

Para la determinación mensual de la incidencia de candidemias se levantaron cohortes retrospectivas, a partir de la información de los Comités de Infecciones. La cohorte está compuesta por todos los pacientes en riesgo (hospitalizados en la UCI), la medida incidente fue el desarrollo de candidemia. La densidad de incidencia (incidencia para el resto del texto) se calculó como: número de casos de candidemias en el mes/número de días/pacientes que estuvieron en riesgo de adquirir la candidemia (estancia en la UCI). También se calculó la densidad de prevalencia (prevalencia de ahora en adelante) como: número de casos de candidemias en el mes/ número de pacientes que estuvieron en riesgo de adquirir la candidemia (egresos de la UCI).

Instituciones: Los participantes fueron: Hospital Universitario San Ignacio, Hospital Universitario Clínica San Rafael, Hospital de Occidente Kennedy, Fundación Hospital San Carlos, Hospital Universitario de la Samaritana, todos en Bogotá, y Hospital Federico Lleras Acosta en Ibagué. Todas las instituciones eran de tercer o cuarto nivel y tenían unidades de cuidado intensivo, así como Comité de Infecciones Intrahospitalarias.

\section{Definiciones}

- Candidemia: Identificación de un microorganismo a partir de al menos un hemocultivo por parte del laboratorio de microbiología del hospital participante. Solamente se contó un episodio por paciente. Para cumplir con este criterio se tomó como válido contar con sólo un hemocultivo positivo. La solicitud de los hemocultivos se realizó en cada institución de acuerdo el criterio de médico tratante del paciente en UCI, siguiendo los protocolos institucionales.

- Estancia en UCI: Número de días de hospitalización en UCI. Este conteo se realizó mensualmente. Los datos se tomaron del Comité de Infecciones Intrahospitalarias de cada institución participante o en otros del servicio de estadística.

- Egreso de UCI: Número de pacientes atendidos mensualmente en la UCI. Los datos se tomaron del Comité de Infecciones Intrahospitalarias de la institución participante o del servicio de estadística.

- En las bases de información de cada institución en formato Whonet, se identificaron las infecciones de torrente sanguíneo por Candida spp., con lo cual se obtuvo la información de frecuencia mensual.

\section{Análisis de la información}

Con la información obtenida de los registros de estadística, del comité de infecciones y de la base de resultados de pruebas de identificación y susceptibilidad bacteriana, se determinó la incidencia de candidemias para cada una de las UCI, para cada uno de los meses del período de observación. Se realizó un análisis descriptivo de las instituciones participantes para cada uno de los años.

Considerando que se propuso la realización de observaciones simultáneas sobre siete series (incidencia de candidemias en UCI para cada institución), utilizando la misma secuencia de puntos en el tiempo, se planteó su análisis mediante modelos univariados de series de tiempo. El tiempo se consideró en meses y se dispuso de 60 períodos comprendidos entre los meses de enero del año 2004 y diciembre del año 2008. Como primer paso, se efectuó una inspección visual del gráfico crudo de la serie para detectar la presencia de tendencia, valores extremos, estacionalidad o discontinuidad. Dependiendo de las características de la serie, se escalaron todas las series para que tuvieran media de cero y varianza de uno (estacionariedad).

Con las observaciones mensuales de la incidencia de C. albicans y $C$. no albicans por 1.000 días/paciente de estancia en UCI, se desarrollaron análisis de series de tiempo para cada una de las instituciones y para la serie agrupada (las siete instituciones). Se utilizaron diferentes filtros de suavizamiento (filtro de Spencer y de promedios móviles de diferentes órdenes), de acuerdo a la longitud de la serie y del número de ceros en ella. Después de evaluar varianza y tendencia, se realizaron las diferentes transformaciones o diferenciaciones requeridas para lograr estacionariedad.

En el análisis estadístico de las series se utilizaron intervalos de confianza de $95 \%$. En lo referente al modelamiento se consideró la opción de incorporar componentes auto-regresivos (AR) o de promedios móviles (MA). Una vez estimados los parámetros de los modelos se efectuaron las pruebas de bondad de ajuste correspondientes. Los análisis se efectuaron con el sistema estadístico SCA. Se calculó también la regresión lineal de la tendencia global.

\section{Resultados}

Durante el período estudiado se obtuvieron los datos de estancia y egresos mensuales en Unidades de Cuidado Intensivo de siete hospitales ( $\mathrm{A} \mathrm{a} \mathrm{G}$ ), así como los reportes de candidemia de los laboratorios de las instituciones participantes. Se obtuvo un total de 382 aislados de Candida spp, repartidas en 214 C. albicans (56\%) y 168 C. no albicans (44\%). En la Tabla 1 se muestran las características de los hospitales participantes, seis de ellos con infectólogo. 
La Tabla 2 muestra la distribución de aislados de especies de Candida y la discriminación por especies en cada uno de los hospitales estudiados. Candida albicans fue aislada en 55,4\% de los casos. Las especies de $C$. no albicans más frecuentemente identificadas fueron C. tropicalis y C. parapsilosis. Los hospitales que aportaron más casos fueron el C (20,7\%), el F (19,6\%) y el A (19,4\%).

Se calculó el total de egresos en UCI y con éste la prevalencia de candidemias para cada institución y la prevalencia total de las mismas. La prevalencia global fue de 1,4\%. La prevalencia al inicio y al final del período estudiado fue $1,8 \%$ y $1,7 \%$, respectivamente, con un valor máximo de 3,5\%. El número de casos de $C$. albicans presentó una prevalencia al comienzo del período de 1,5\% y $1,0 \%$ final del mismo, con un valor máximo de $1,6 \%$. Se registró un valor de $C$. no albicans de $0,3 \%$ al inicio del período y de $0,6 \%$, con un valor máximo de $2,2 \%$. Al analizar individualmente los gráficos de las series de tiempo de cada hospital, se observó un incremento en el número de casos en los años 2005 y 2007 en el hospital E, en el 2007 en el hospital F, y en 2005 en el hospital C. En el hospital A se observan incrementos aislados esporádicos con mayor frecuencia en el periodo estudiado. En general, se observó un aumento en la prevalencia total de candidemias (Figura 1) a expensas de casos de $C$. no albicans (incremento de 0,0016 casos por mes, $\mathrm{p}<0,05$ ).

La incidencia global fue de 2,3 casos por 1.000 días de UCI. La incidencia al inicio y al final del período estudiado fue 2,5 con un valor máximo de 5,1 por cada 1.000 días de estancia. La incidencia global de C. albicans fue de 1,3 casos por 1.000 días de UCI, con un una incidencia al inicio del período de 2,2 casos por 1.000 días de hospitalización y de 1,5 casos por 1.000 días de UCI al final del mismo, con un valor máximo de 3,0. La incidencia global de $C$. no albicans fue de 1,0 casos por 1.000 días de hospitalización, con 0,4 casos por 1.000 días de UCI al inicio del período y de 0,9 al final del período estudiado con un valor máximo de 3,1 . Se observó una tendencia al aumento de incidencia de candidemias con un descenso en los casos de candidemias por C. albicans y un incremento compensatorio de casos de $C$. no albicans (Figura 2), incremento mensual de las candidemias de 0,0032 , la disminución mensual de $C$. albicans de 0,0034 e incre-

Tabla 1. Características de los hospitales participantes

\begin{tabular}{clccc} 
Institución & Servicio & $\begin{array}{c}\text { n de } \\
\text { camas }\end{array}$ & $\begin{array}{c}\text { n de días } \\
\text { de estancia } \\
\text { promedio }\end{array}$ & $\begin{array}{c}\text { n de egresos } \\
\text { promedio } \\
\text { al mes }\end{array}$ \\
A & UCl Cardiología adultos & 24 & 4 & 150 \\
& UCl médica adulto & 12 & 5,3 & 53 \\
& UCl coronaria & 15 & 2 & 160 \\
& UCl pediátrica & 8 & 7 & $25-30$ \\
& UCl pediátrica cardiovascular & 12 & 5 & $50-60$ \\
& UCl neonatal & 14 & 10,5 & $45-50$ \\
B & UCl & 24 & 7,6 & 35 \\
C & Adultos & 15 & 21 & $25-38$ \\
& Intermedia & 7 & 14 & $20-30$ \\
D & Adulto Unidad 1 & 16 & 8 & 490 \\
& Coronario Unidad & 12 & 8 & 399 \\
& Adulto Unidad 2 & 10 & 5 & 480 \\
& Adulto Unidad 3 & 10 & 6 & 386 \\
E & UCl & 28 & 6,5 & 125 \\
& UCl neonatal & 32 & - & - \\
F & UCl & 43 & 7 & 163 \\
& UCl pediátrica & 10 & - & 27 \\
G & UCl & 11 & 8 & 35 \\
\hline
\end{tabular}

Tabla 2. Distribución de aislados de Candida por especies en los hospitales participantes

\begin{tabular}{|c|c|c|c|c|c|c|c|c|}
\hline $\begin{array}{l}\text { Aislados de Candida por } \\
\text { especies }\end{array}$ & $\begin{array}{l}\text { Hospital A } \\
\text { n (\%) }\end{array}$ & $\begin{array}{l}\text { Hospital B } \\
\text { n (\%) }\end{array}$ & $\begin{array}{c}\text { Hospital C } \\
\text { n (\%) }\end{array}$ & $\begin{array}{l}\text { Hospital D } \\
\text { n (\%) }\end{array}$ & $\begin{array}{l}\text { Hospital E } \\
\text { n (\%) }\end{array}$ & $\begin{array}{l}\text { Hospital F } \\
\text { n (\%) }\end{array}$ & $\begin{array}{l}\text { Hospital G } \\
\text { n (\%) }\end{array}$ & $\begin{array}{l}\text { Aislados } \\
\text { n (\%) }\end{array}$ \\
\hline Candida albicans & $33(44,6)$ & $6(42,8)$ & $50(63,3)$ & $21(43,8)$ & $42(62,7)$ & $51(68)$ & $11(44)$ & $214(56,0)$ \\
\hline C. tropicalis & $15(20,3)$ & $2(14,2)$ & $7(8,9)$ & $18(37,5)$ & $12(17,9)$ & $8(10,6)$ & $4(16)$ & $66(17,3)$ \\
\hline C. parapsilosis & $18(24,3)$ & $2(14,2)$ & $12(15,2)$ & $6(12,5)$ & $10(14,9)$ & $8(10,6)$ & $5(20)$ & $61(16,0)$ \\
\hline C. glabrata & $2(2,7)$ & 0 & 0 & $2(4,1)$ & $3(4,4)$ & 0 & $3(12)$ & $10(2,6)$ \\
\hline C. Iusitaniae & $2(2,7)$ & 0 & 0 & 0 & 0 & $1(1,3)$ & $1(4)$ & $4(1,1)$ \\
\hline C. krusei & $3(4,0)$ & 0 & 0 & 0 & 0 & 0 & 0 & $3(0,8)$ \\
\hline Otras Candidas identificadas & $1(1,3)$ & 0 & $9(11,3)$ & $1(2,1)$ & 0 & $6(8)$ & 0 & $17(4,1)$ \\
\hline Candida spp.* & 0 & $4(28,6)$ & $1(1,2)$ & 0 & 0 & $1(1,3)$ & $1(4)$ & $7(1,8)$ \\
\hline Total aislados & $74(19,4)$ & $14(3,6)$ & $79(20,7)$ & $48(12,5)$ & $67(17,5)$ & $75(19,6)$ & $25(6,5)$ & 382 \\
\hline
\end{tabular}


mento de candidemias no albicans de 0,0066, $\mathrm{p}<0,05$ ). En el análisis por institución, se observó un incremento en el número de casos en los años 2005 y 2007 en el hospital E, en 2005 en el hospital C, y de forma notable en los años 2006 y 2007 en el hospital F. En el hospital A se observaronn incrementos aislados esporádicos con mayor frecuencia en el período estudiado.

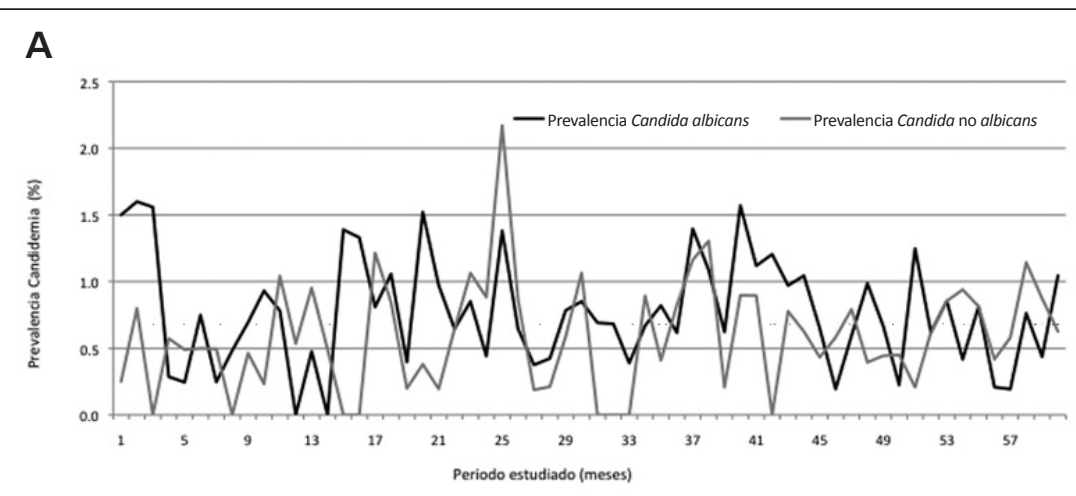

B

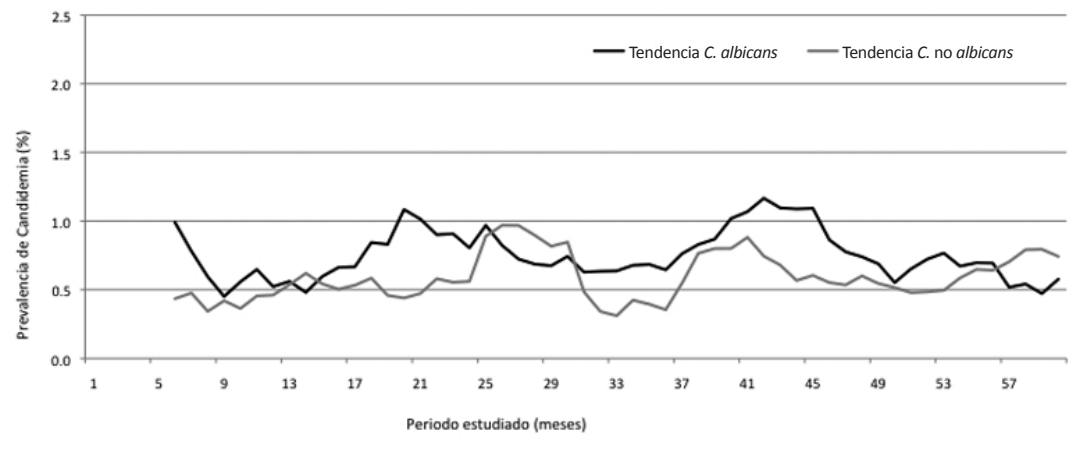

Figura 1. Prevalencia del total de candidemias durante el período estudiado y la línea de tendencia en promedios móviles de seis meses.

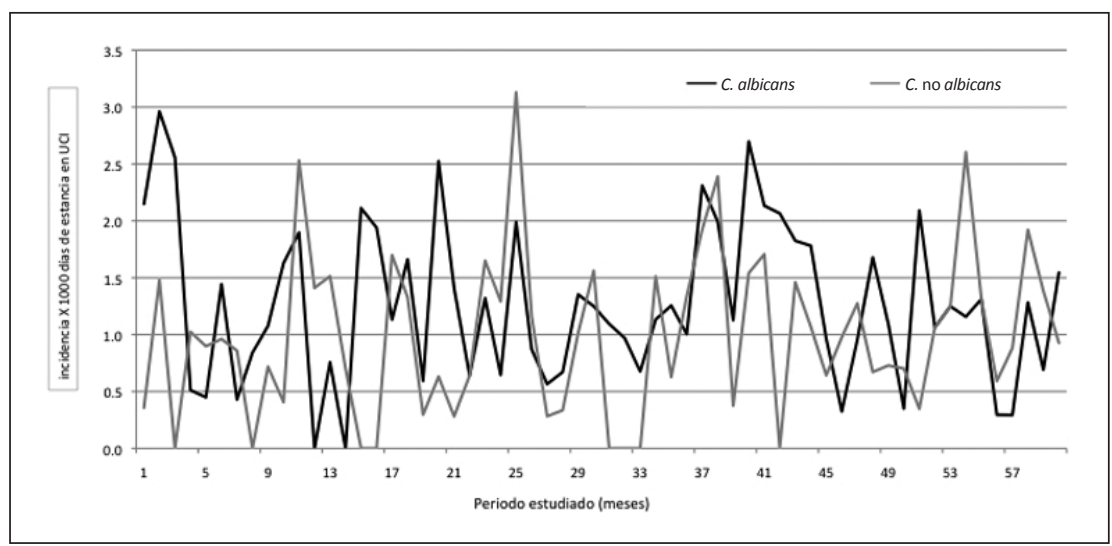

Figura 2. Incidencia del total de candidemias durante el período estudiado y la línea de tendencia en promedios móviles de seis meses.

\section{Discusión}

Este estudio muestra la incidencia y prevalencia de candidemia en UCI de siete hospitales colombianos. Se encontró una prevalencia global en estas unidades de las hospitales de $1,4 \%$, y una incidencia de 2,3 por 1.000 días de estancia hospitalaria, cifra significativamente mayor a lo reportado en Brasil y países desarrollados como Países Bajos, Suiza y E.U.A., en los que se suele observar una incidencia $<1$ caso por 1.000 días de estancia hospitalaria $^{12,13}$. La prevalencia identificada en Colombia también es superior a la identificada en el estudio EPIC II, que recogió pacientes en UCIs de 76 países en los cinco continentes, identificando una prevalencia de $0,69 \%{ }^{14}$.

¿Cuáles pueden ser las razones que expliquen una incidencia mucho mayor a la observada en países desarrollados? Se han descrito ciertos factores de riesgo que determinan el aumento de casos de candidemias. Entre ellos están los procedimientos invasores (catéteres), ventilación mecánica y estancia hospitalaria ${ }^{13}$. Estos factores de riesgo se encuentran con mayor frecuencia en las UCIs de los países en vías de desarrollo ${ }^{15,16}$. El uso de dispositivos invasores (catéteres venosos centrales, catéteres urinarios, ventilación mecánica) es un factor fuertemente vinculado con infecciones relacionadas al cuidado de la salud. Su uso y la incidencia de las infecciones asociadas a dispositivos son más altas en América Latina. En Colombia, Moreno y cols., informó una tasa de infecciones asociadas a catéter central de 11,3 por 1.000 catéteres/días de internación en hospitales en Colombia ${ }^{15}$. El International Nosocomial Infection Control Consortium (INICC) reportó en 2008 datos sobre infecciones asociadas a dispositivos del cuidado de la salud en países en vías de desarrollo y lo comparó con el sistema norteamericano del National Healtcare Safety Network (NHSN, previamente conocido como National Nosocomial Infection System- NNIS). Estos datos muestran que la tasa de infección asociada a dispositivos, usualmente neumonía asociada a ventilación y bacteriemia asociada al catéter, son un problema serio, documentándose mayores tasas en los países en vías de desarrollo como Colombia ${ }^{16,17}$. Adicionalmente, estos estudios han señalado cómo los pacientes en cuidado intensivo requieren mayor uso de dispositivos invasores en comparación a lo identificado en países desarrollados. Ello significa que los pacientes se encuentran cada vez más tiempo con riesgo de desarrollar candidemia por mayor nivel de exposición a estos factores de riesgo.

Otra razón por la cual la incidencia y prevalencia de candidemia son más elevadas en Colombia podría ser el impacto de la transmisión cruzada en UCI. La razón entre camas de UCI y personal de enfermería, y el lavado de manos son factores independientes que tienen un alto impacto en la incidencia de infecciones asociadas al cuidado de la salud $^{18,19}$. El estudio INICC y otros han mostrado la baja 
aplicación del lavado de manos en nuestro medio ${ }^{20}$. Esto es importante, ya que se reconoce con mayor frecuencia la posibilidad de transmisión cruzada de Candida spp en las $\mathrm{UCI}^{21,22}$. Los datos de incrementos observados durante periodos de tiempo en los hospitales podrían representar brotes en las instituciones; sin embargo, en ausencia de confirmación epidemiológica o molecular, no se puede establecer con certeza. En Colombia sí se han reportado brotes de candidemia en UCI neonatal ${ }^{23}$.

Otro de los aspectos importantes en la candidemia ha sido el cambio epidemiológico a expensas de un aumento en los casos de $C$. no albicans. En este estudio se observa esta tendencia en los hospitales estudiados así como han sido reportados en España, Bélgica y Turquía ${ }^{2,4,24}$. El uso temprano o profiláctico de antifúngicos de la familia de los azoles, específicamente fluconazol, ha sido asociado al cambio de patrón epidemiológico. Abi-Said y cols., reportaron una disminución de casos de C. albicans y C. tropicalis, con un aumento de casos de C. glabrata y C. krusei tras el uso de fluconazol ${ }^{25}$. No disponemos de información sobre consumo de antifúngicos para entender las razones de este cambio, especialmente cuando no se ha observado en esta serie un incremento notorio en la frecuencia de C. krusei o C. glabrata, especies claramente seleccionadas por el uso de fluconazol.

El incremento de especies de $C$. no albicans, y con ellas de patrones de resistencia a antifúngicos puede asociarse con desenlaces no deseados ${ }^{26}$. Las $C$. no albicans pueden estar relacionadas con resistencia a fluconazol; particularmente $C$. glabrata y $C$. krusei se han asociado a este problema $^{27}$. También se ha reportado este fenómeno en $C$. tropicalis y $C$. parapsilosis (especies de $C$. no albicans más frecuentes en este estudio) en reportes recientes de Latinoamérica y el mundo. Pappas y cols., reportaron una mayor tasa de mortalidad atribuible en aquellos pacientes con C. albicans ${ }^{28}$. Esta discusión es muy importante a la hora de escoger la terapia empírica en los pacientes con sospecha de candidemia. Datos recientes de susceptibilidad en América Latina muestran una alta susceptibilidad a fluconazol y, como en nuestro estudio, una alta frecuencia de $C$. parapsilosis, por lo que la recomendación de primera línea de tratamiento no es clara ${ }^{29}$.

Nuestro trabajo tiene importantes limitaciones. Éste es un estudio de prevalencia retrospectivo, en el que los datos recolectados provienen de los laboratorios de microbiología y de los comités de infección intrahospitalaria de las instituciones participantes. En estas instituciones no se realizaban pruebas de susceptibilidad para la época del estudio. Se desconocen datos como exposición a factores de riesgo mencionados, ni cambios en la frecuencia de diversos grupos inmunosuprimidos (por ejemplo pacientes con cáncer) y no se puede establecer relación causa-efecto entre los cambios epidemiológicos y el uso de azoles en nuestro medio. Por otro lado, teniendo la baja sensibilidad de los hemocultivos, la incidencia y prevalencia real de candidemia puede estar subestimada.

Agradecimientos: A las instituciones participantes, así como a los miembros colaboradores en cada institución: Hospital Universitario San Ignacio (Carlos Hernando Gómez), Hospital Universitario Clínica San Rafael (Carlos Saavedra), Hospital de Occidente Kennedy (Elkin Lemos), Fundación Hospital San Carlos (Jaime Saravia), Hospital Universitario de la Samaritana (Lucy Guzmán), Hospital Federico Lleras Acosta (Amparo Ovalle Garzón). Agradecemos también a Anita Montañez, coordinadora de investigación del Grupo de Investigación en Enfermedades Infecciosas de la Universidad Nacional de Colombia por su apoyo logístico y a Andrés Meneses, por el apoyo con las bases de datos.

\section{Resumen}

Introducción: La candidemia es una complicación con alta morbilidad y mortalidad en pacientes en cuidado intensivo. Objetivo: Determinar la incidencia y prevalencia de candidemia en hospitales colombianos entre 2004 y 2008. Materiales y Métodos: En siete hospitales colombianos se obtuvieron retrospectivamente los datos de candidemia confirmada por el laboratorio y de estancia y egreso en unidades de cuidado intensivo. Se construyeron series de tiempo de densidad de incidencia (definida como el número de casos de candidemias en el mes en relación al número de días-pacientes hospitalizados) y de densidad de prevalencia mensuales (número de casos de candidemias en el mes en relación al número de egresos) para cada institución y para el conjunto de hospitales. Resultados: Se identificaron 382 casos, con una incidencia de 2,3 casos por 1.000 días de estancia en UCI, y una prevalencia fue de $1,4 \%$. Se observó una tendencia al aumento en la incidencia $(0,0066$ casos adicionales por cada 1.000 días de estancia por mes) y en la prevalencia de candidemia $(0,0016$ casos adicionales por 100 pacientes por mes). El aumento en la prevalencia y en la incidencia se observó a partir de un incremento en las especies de Candida no albicans, la cual correspondió a 44\% de los aislados totales. Discusión y conclusiones: La incidencia y la prevalencia de candidemia en Colombia son elevadas y van en aumento, especialmente a expensas de casos de Candida no albicans. 


\section{Referencias bibliográficas}

1.- Garbino J, Kolarova L, Rohner P, Lew D, Pichna P, Pittet D. Secular trends of candidemia over 12 years in adult patients at a tertiary care hospital. Medicine (Baltimore) 2002; 81: 425-33.

2.- Almirante B, Rodríguez D, Park B J, Cuenca-Estrella M, Planes A M, Almela M, et al. Epidemiology and predictors of mortality in cases of Candida bloodstream infection: results from population-based surveillance, Barcelona, Spain, from 2002 to 2003. J Clin Microbiol 2005; 43 : 1829-35.

3.- Colombo A L, Nucci M, Park B J, Nouer S A, Arthington-Skaggs B, da Matta D A, et al. Epidemiology of candidemia in Brazil: a nationwide sentinel surveillance of candidemia in eleven medical centers. J Clin Microbiol 2006; 44: 2816-23.

4.- Erdem I, Oguzoglu N, Ozturk Engin D, Ozgultekin A, Inan A S, Ceran N, et al. Incidence, etiology and risk factors associated with mortality of nosocomial candidemia in a tertiary care hospital in Istanbul, Turkey. Med Princ Pract 2010; 19: 463-7.

5.- Cortés J A, Reyes P, Gómez C, Buitrago G, Leal A L. Fungal bloodstream infections in tertiary care hospitals in Colombia. Rev Iberoam Micol 2011; 28: 74-8.

6.- Gudlaugsson O, Gillespie S, Lee K, Vande Berg J, Hu J, Messer S, et al. Attributable mortality of nosocomial candidemia, revisited. Clin Infect Dis 2003; 37: 1172-7.

7.- Horn D L, Neofytos D, Anaissie E J, Fishman J A, Steinbach W J, Olyaei A J, et al. Epidemiology and outcomes of candidemia in 2019 patients: data from the prospective antifungal therapy alliance registry. Clin Infect Dis 2009; 48: 1695-703.

8.- $\quad$ Trick W E, Fridkin S K, Edwards J R, Hajjeh R A, Gaynes R P. Secular trend of hospital-acquired candidemia among intensive care unit patients in the United States during 1989-1999. Clin Infect Dis 2002; 35: 627-30.

9.- Blumberg H M, Jarvis W R, Soucie J M, Edwards J E, Patterson J E, Pfaller M A, et al. Risk factors for candidal bloodstream infections in surgical intensive care unit patients: the NEMIS prospective multicenter study. The National Epidemiology of Mycosis Survey. Clin Infect Dis 2001; 33: 177-86.
10.- Snydman D R. Shifting patterns in the epidemiology of nosocomial Candida infections. Chest 2003; 123: 500S-3S.

11.- Henríquez D, Ardila E, Leal A L, Castillo J S, Buitrago G, Bermúdez D, et al, Manual para la toma de muestras para análisis microbiológico, 2006.

12.- Girao E, Levin A S, Basso M, Gobara S, Gomes L B, Medeiros E A, et al. Seven-year trend analysis of nosocomial candidemia and antifungal (fluconazole and caspofungin) use in Intensive Care Units at a Brazilian University Hospital. Med Mycol 2008; 46: 581-8.

13.- Eggimann P, Garbino J, Pittet D. Epidemiology of Candida species infections in critically ill non-immunosuppressed patients. Lancet Infect Dis 2003; 3: 685-702.

14.- Kett D H, Azoulay E, Echeverria P M, Vincent J L. Candida bloodstream infections in intensive care units: analysis of the extended prevalence of infection in intensive care unit study. Crit Care Med 2011; 39: 665-70.

15.- Moreno C A, Rosenthal V D, Olarte N, Gomez W V, Sussmann O, Agudelo J G, et al. Device-associated infection rate and mortality in intensive care units of 9 Colombian hospitals: findings of the International Nosocomial Infection Control Consortium. Infect Control Hosp Epidemiol 2006; 27: 349-56.

16.- Rosenthal V D, Maki D G, Mehta A, Álvarez-Moreno C, Leblebicioglu $\mathrm{H}$, Higuera F, et al. International Nosocomial Infection Control Consortium report, data summary for 2002-2007, issued January 2008. Am J Infect Control 2008; 36: 627-37.

17.- Dudeck M A, Horan T C, Peterson K D, Allen-Bridson K, Morrell G C, Pollock D A, et al. National Healthcare Safety Network (NHSN) report, data summary for 2009, device-associated module. Am J Infect Control 39: 349-67.

18.- Pittet D, Allegranzi B, Sax H, Dharan S, Pessoa-Silva C L, Donaldson L, et al. Evidencebased model for hand transmission during patient care and the role of improved practices. Lancet Infect Dis 2006; 6: 641-52.

19.- Stone P W, Pogorzelska M, Kunches L, Hirschhorn L R. Hospital staffing and health care-associated infections: a systematic review of the literature. Clin Infect Dis 2008; 47: 937-44.

20.- Barrera L, Zingg W, Méndez F, Pittet D. Effectiveness of a hand hygiene promotion strategy using alcohol-based handrub in 6 intensive care units in Colombia. Am J Infect Control 2011; 39: 633-9.

21.- Asmundsdottir L R, Erlendsdottir H, Haraldsson G, Guo H, Xu J, Gottfredsson M. Molecular epidemiology of candidemia: evidence of clusters of smoldering nosocomial infections. Clin Infect Dis 2008; 47: e17-24.

22.- Chen K W, Chen Y C, Lin Y H, Chou H H, Li S Y. The molecular epidemiology of serial Candida tropicalis isolates from ICU patients as revealed by multilocus sequence typing and pulsed-field gel electrophoresis. Infect Genet Evol 2009; 9: 912-20.

23.- Díaz Granados C A, Martínez A, Deaza C, Valderrama S. An outbreak of Candida spp. bloodstream infection in a tertiary care center in Bogota, Colombia. Braz J Infect Dis 2008; 12: 390-4

24.- Swinne D, Watelle M, Suetens C, Mertens K, Fonteyne PA, Nolard N. A one-year survey of candidemia in Belgium in 2002. Epidemiol Infect 2004; 132: 1175-80.

25.- Abi-Said D, Anaissie E, Uzun O, Raad I, Pinzcowski H, Vartivarian S. The epidemiology of hematogenous candidiasis caused by different Candida species. Clin Infect Dis 1997; 24: 1122-8.

26.- Pfaller M A, Messer S A, Moet G J, Jones R N, Castanheira M. Candida bloodstream infections: comparison of species distribution and resistance to echinocandin and azole antifungal agents in Intensive Care Unit (ICU) and nonICU settings in the SENTRY Antimicrobial Surveillance Program (2008-2009). Int J Antimicrob Agents 2011; 38: 65-9.

27.- Pfaller M A, Diekema D J, Gibbs D L, Newell V A, Barton R, Bijie H, et al. Geographic variation in the frequency of isolation and fluconazole and voriconazole susceptibilities of Candida glabrata: an assessment from the ARTEMIS DISK Global Antifungal Surveillance Program. Diagn Microbiol Infect Dis; 67: 162-71.

28.- Pappas P G, Rex J H, Lee J, Hamill R J, Larsen R A, Powderly W, et al. A prospective observational study of candidemia: epidemiology, therapy, and influences on mortality in hospitalized adult and pediatric patients. Clin Infect Dis 2003; 37: 634-43.

29.- Nucci M, Queiroz-Telles F, Alvarado-Matute T, Tiraboschi IN, Cortés J, Zurita J, et al. Epidemiology of candidemia in Latin America: a laboratory-based survey. PLoS One 2013; 8: e59373. 